\title{
Dosimétrie passive des neutrons rapides au moyen des diodes de silicium
}

\author{
F. SPURNY*,**, R. MEDIONI*, L. PLAWINSKI*, Q. CHAU*
}

(Manuscrit reçu le 9 janvier 1999, révisé le 16 juillet 1999, accepté le 22 septembre 1999)

RÉSUMÉ Les diodes de silicium sont utilisées depuis plusieurs années pour la dosimétrie des neutrons rapides dans le cas d'irradiation à de fortes doses, y compris les irradiations accidentelles. Cet article décrit les résultats des tests effectués sur des diodes d'une nouvelle fabrication dont il présente également une analyse de leurs caractéristiques et des possibilités d'utilisation. Des diodes de deux sensibilités différentes ont été irradiées auprès du réacteur SILENE (IPSN Valduc) ce qui a permis d'étudier: la reproductibilté des lectures ainsi que l'influence de leur répétition, l'homogénéité des lots de diodes étudiées, l'évolution du signal en fonction du temps après l'irradiation, l'influence du traitement thermique après irradiation sur cette évolution, la réponse de deux types de diodes testés aux neutrons du réacteur SILENE dans deux configurations différentes, le seuil de détection exprimé en kerma dû aux neutrons dans le tissu. Les résultats obtenus sont presentés, analysés et discutés. Enfin, les principales connaissances acquises sont résumées dans la conclusion.

ABSTRACT Fast neutrons passive dosimetry with Si-diodes.

Since several years, Si-diodes have been used for the fast neutron dosimetry in the case of high level exposure, accidental enclosed. This work describes the results of tests performed with the diodes of a new production, presents their characteristics and the analysis of the possibilities of their use. The diodes of two different sensitivities were irradiated at the SILENE reactor (IPSN Valduc). We studied: the reproducibility of reading and the influence of its repetition, the homogeneity of the lot of diodes studied, the evolution of the signal with the time after irradiation, the influence of the thermal treatment on this evolution, the response of two types of diodes to the neutrons of SILENE reactor in two configurations, the detection threshold expressed in tissue kerma. The results obtained are presented, analysed and discussed. The principal knowledge is formulated in the conclusion.

\section{Introduction}

Les diodes de silicium sont utilisées depuis plusieurs années pour la dosimétrie des neutrons rapides dans le cas d'irradiation forte (Blanc, 1990 ; Delafield and Reading,

\footnotetext{
* IPSN, Institut de protection et de sûrete nucléaire, departement de protection de la santé de l'homme et de dosimetrie, service de dosimetrie, BP n ${ }^{\circ} 6,92265$ - Fontenay-aux-Roses Cedex, France

** Present Address: Académie des sciences de la République Tchèque, département de dosimétrie, institut de physique nucléaire, Na Truhlarce 39/64, 18086 Praha 8, Républiquc Tchèque.
} 
1981). Au service de dosimétrie (SDOS) du département de protection de la santé de l'homme et de dosimétrie de l'IPSN, ces diodes ont été utilisées depuis plusieurs années pour la dosimétrie d'accident de criticité ainsi que pour la dosimétrie neutrons en radiobiologie. Récemment, nous avons fait l'acquisition de diodes en provenance de la République Tchèque (Prouza et al., 1989 ; Skubal, 1981). Cet article décrit les résultats des tests de ces diodes et présente leur analyse.

\section{Matériel et méthodes}

\subsection{Diodes testées}

Les principales caractéristiques des deux types de diodes fournis par le fabricant sont presentées dans le tableau I.

Deux séries de tests ont été effectuées. Nous avons utilisé 30 diodes de chaque type pour la première série, 50 diodes de chaque type pour la deuxième. Les tensions de départ étaient: $\mathrm{Si}-1:(1023 \pm 17) \mathrm{mV}$ ou $(1028 \pm 14) \mathrm{mV}$, et $\mathrm{Si}-2:(2143$ $\pm 39) \mathrm{mV}$, ou (2 142 \pm 74$) \mathrm{mV}$, pour la série No. 1, ou No. 2, en accord avec les valeurs du tableau I.

\subsection{Irradiation}

L'irradiation des diodes a été effectuée au cours de trois tirs du réacteur SILENE (Tournier et al., 1997), deux fois avec le réacteur sous $10 \mathrm{~mm}$ de $\mathrm{Pb}$, une fois avec le réacteur nu. Les trois tirs ont été réalisés en mode de libre évolution, le nombre de fissions s'établissant respectivement à $3,04 \times 10^{17}, 9,2 \times 10^{16}$ et $1,10 \times 10^{17}$. Les diodes ont été irradiées à des distances comprises entre 3 et $7 \mathrm{~m}$ du centre du coeur du réacteur, dans l'air pour le réacteur nu, dans l'air et sur fantôme pour le réacteur sous plomb.

Trois diodes de chaque type étaient, pour les deux séries, gardées comme témoins.

\subsection{Traitement des diodes avant et au cours de l'évaluation}

Toutes les diodes ont été, après l'irradiation, traitées de la façon suivante :

1) une partie des diodes a été chaufée dans un four à $100^{\circ} \mathrm{C}$ pendant soit une heure, soit 30 minutes;

2) quelques diodes ont été plongées dans l'eau bouillante pendant 3 minutes;

3) un tiers de diodes de chaque série n'a pas reçu de traitement thermique;

4) toutes les diodes (témoins compris) testées dans la première série ont été, de plus, chauffées, 78 jours après irradiation, dans un four à $100^{\circ} \mathrm{C}$ pendant une heure. 
TABLEAU I

Caractéristiques principales des deux types de diodes testées. Basic characteristics of two types of diodes tested.

\begin{tabular}{|lcc|}
\hline & \multicolumn{2}{c|}{ Type } \\
\hline Gamme des doses & $\mathrm{Si}-1$ & $\mathrm{Si}-2$ \\
\hline Réponse aux neutrons ${ }^{252} \mathrm{Cf}$ & $0.1-30 \mathrm{~Gy}$ & $0,01-5 \mathrm{~Gy}$ \\
Tension de départ $(\mathrm{D}=0)$ & $0,13 \mathrm{~V} / \mathrm{Gy}( \pm 20 \%)$ & $1,1 \mathrm{~V} / \mathrm{Gy}( \pm 20 \%)$ \\
\hline Augmentation de la tension avec la température & $1030 \mathrm{mV}( \pm 5 \%)$ & $2100 \mathrm{mV}( \pm 5 \%)$ \\
\hline
\end{tabular}

\section{4. Évaluation des diodes}

Le principe de fonctionnement des diodes repose sur les défauts stables créés par les neutrons qui modifient de manière permanente la structure de la diode. Ces défauts mènent au changement du temps de vie des porteurs, ceci entraine une augmentation de la tension sur la diode pour un courant constant. La réponse est donc representée par la différence entre la tension avant et après irradiation. Cette différence est la fonction de la dose absorbée dans le silicium, elle est aussi la fonction du temps écoulé depuis l'irradiation et du traitement thermique de chacune des diodes.

Les diodes ont été évaluées au moyen d'un lecteur FIMEL (type L.D.N.). La lecture a été répetée pendant 90 jours dans le cas de la première série, et pendant 57 jours pour la deuxième. La température ambiante n’a pas été spécialement suivie et/ou enregistrée.

Il est apparu que la tension mesurée change avec la répétition des lectures, surtout pour les diodes plus sensibles (type $\mathrm{Si}$-2). Il faut 5 à 6 lectures successives sur la même diode pour que la tension se stabilise. Les résultats des lectures stabilisées ainsi obtenus ont été comparés à ceux obtenus pour une lecture unique, les conclusions sont presentées plus loin.

\section{Résultats et analyse}

\subsection{Reproductibilité des lectures}

La réproductibilité d'une lecture peut être analysée à partir des lectures des diodes témoins effectuées pendant 90 jours après irradiation. Elle est environ de $0,2 \%$ pour $\mathrm{Si}-1$, et de $0,3 \%$ pour le $\mathrm{Si}-2$ et peut être considéré comme bonne si on tient 
compte de la période de lecture et des variations de la témperature ambiante possibles. Il faut rappeler qu'en absolu, une variation de $0,1 \%$ correspond pour Si-1 à une différence de la tension de $1 \mathrm{mV}$ et pour $\mathrm{Si}-2$ de $2 \mathrm{mV}$.

\subsection{Influence de la répétition des lectures}

L'application d'un courant aux bornes de la diode change la tension. Quand on répète la mesure, avec la même diode, la tension affichée augmente. Cette augmentation dépend du type de diode, et se stabilise après 5 à 6 mesures consecutives. Pour les diodes-témoins, cet effet $(+0,1 \%$ pour $\mathrm{Si}-1,+0,3 \%$ pour Si-2), n'est pas significatif par rapport à la reproductibilité des lectures, alors que pour les diodes irradiées il est plus conséquant. Son amplitude est inférieure à $1 \%$ pour le Si-1, mais autour de $4 \%$ pour le Si-2. Il faut donc traiter séparemment les résultats de la lecture unique et ceux de la lecture après stabilisation pour le type $\mathrm{Si}-2$.

\subsection{Homogénéité des lots de diodes testées}

L'analyse montre que, pour juger l'homogéneité des lots de diodes, il faut réaliser les lectures au moins 40 jours après irradiation, donc suffissamment eloignés du moment de l'irradiation et du traitement thermiques qui s'en suivait. Les différences

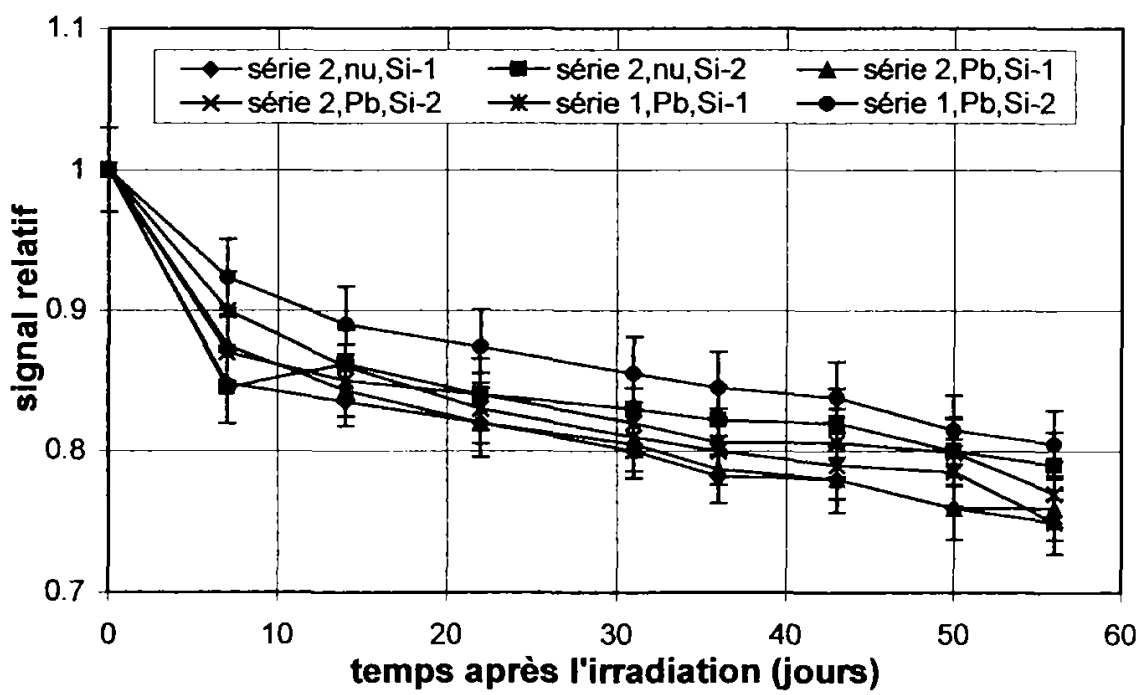

Fig. 1. - «Fading » des diodes thermiquement non traitées.

Fading of thermally non treated diodes. 


\section{TABLEAU II}

Réponses moyennes des diodes chauffées aprés irradiation $\left(100^{\circ} \mathrm{C}, 1\right.$ heure); $1^{\text {re }}$ série, période de lecture entre 0,25 et 90 jours.

Average responses of diodes heated $\left(100^{\circ} \mathrm{C}, 1\right.$ hour $)$ after the irradiation; 1 st serie, reading intervall between 0.25 and 90 days.

\begin{tabular}{|c|c|c|c|c|}
\hline \multirow{3}{*}{ Type } & \multicolumn{4}{|c|}{ Réponse moyenne $\Delta \mathbf{U}, \mathbf{m V}$, des diodes irradiées à } \\
\hline & \multicolumn{2}{|c|}{$4 \mathrm{~m}$} & \multicolumn{2}{|c|}{$7 \mathrm{~m}$} \\
\hline & Lecture unique & Lecture stabilisée & Lecture unique & Lecture stabilisée \\
\hline $\mathrm{Si}-1$ & $517,0 \pm 4,4$ & $522,1 \pm 4,4$ & $177,5 \pm 0,9$ & $178,2 \pm 0,8$ \\
\hline $\mathrm{Si}-2$ & $3208 \pm 40$ & $3321 \pm 45$ & $1231 \pm 22$ & $1282 \pm 22$ \\
\hline
\end{tabular}

TABLEAU III

Réponses moyennes des diodes chauffées après irradiation $\left(100^{\circ} \mathrm{C}\right), 2^{\mathrm{e}}$ série, période de lecture entre 0,25 et 57 jours, $1^{\text {re }}$ lecture.

Average responses of diodes heated $\left(100^{\circ} \mathrm{C}\right)$ after the irradiation, 2nd serie, reading intervall between 0.25 and 57 days, 1st reading.

\begin{tabular}{|c|c|c|c|c|}
\hline \multirow{3}{*}{ Type } & \multicolumn{4}{|c|}{ Réponse moyenne $\Delta U,[\mathrm{mV}]$, des diodes irradiées auprès de } \\
\hline & \multicolumn{2}{|c|}{ SII.ENE/Pb } & \multicolumn{2}{|c|}{ SIL.ENE nu } \\
\hline & chauffée $1 / 2$ h & chauffée $1 \mathrm{~h}$ & chauffée $1 / 2$ h & chauffée 1 h \\
\hline $\mathrm{Si}-1$ & $241,6 \pm 0,6$ & $229,2 \pm 0,4$ & $325,3 \pm 2,2$ & $311,5 \pm 2,1$ \\
\hline $\mathrm{Si}-2$ & $1732 \pm 22$ & $1672 \pm 20$ & $2247 \pm 63$ & $2203 \pm 38$ \\
\hline
\end{tabular}

individuelles de réponse pour le type $\mathrm{Si}-1$ sont d'environ 3,5 à $5 \%$, et pour le type $\mathrm{Si}-2$ entre 10 et $15 \%$, indépendamment du mode de lecture.

\subsection{Réponse en fonction du temps après irradiation, influence du traitement thermique}

La réponse des diodes n'ayant pas subi de traitement thermique diminue régulièrement avec le temps après irradiation, indépendamment du mode de lecture. Les résultats obtenus sont présentés pour les deux séries des tests sur la figure 1.

On voit que cette diminution est assez rapide pendant les 15 premiers jours, puis plus lente, de l'ordre de $20 \%$ au bout de 60 jours. Par contre, la réponse des diodes chauffées à $100^{\circ} \mathrm{C}$ reste, pendant la même periode, pratiquement constante. Les valeurs moyennes typiques sont présentées dans les tableaux II et III. 


\section{TABLEAU IV}

Influence du traitement thermique sur la réponse peu de temps après l'irradiation. Influence of the thermal treatment on the response early after the irradiation.

\begin{tabular}{|cccc|}
\hline Type & Traitement & Réponse relative suivant le temps après irradiation \\
& & 3 heures & 7 jours \\
\hline \multirow{2}{*}{$\mathrm{Si-1}$} & aucun & $1,00^{(1)}$ & $0,87 \pm 0,05$ \\
& ébullition & $0,82 \pm 0,04$ & $0,80 \pm 0,04$ \\
& four, $100^{\circ} \mathrm{C}, 1 / 2 \mathrm{~h}$ & $0,71 \pm 0,03$ & $0,70 \pm 0,03$ \\
& four, $100^{\circ} \mathrm{C}, 1 \mathrm{~h}$ & $0,69 \pm 0,03$ & $0,68 \pm 0,03$ \\
\hline \multirow{2}{*}{$\mathrm{Si}-2$} & aucun & $1,00^{(1)}$ & $0,89 \pm 0,04$ \\
& ébullition & $0,83 \pm 0,04$ & $0,78 \pm 0,04$ \\
& four, $100^{\circ} \mathrm{C}, 1 / 2 \mathrm{~h}$ & $0,73 \pm 0,04$ & $0,70 \pm 0,03$ \\
\hline & four, $100^{\circ} \mathrm{C}, 1 \mathrm{~h}$ & $0,71 \pm 0,03$ & $0,68 \pm 0,03$ \\
\hline
\end{tabular}

(1) valeur relative de réference

On constate que les écarts-types relatifs sont faibles et que ce type de traitement diminue légèremment la réponse. En ce qui concerne les diodes «bouillies » dans l'eau pendant 3 minutes, leur réponse n'est pas tout à fait stable. Elle diminue au bout de 80 jours de $6 \%$ pour le type $\mathrm{Si}-1$, et de $10 \%$ pour le type $\mathrm{Si}-2$.

Néanmoins, il faut rappeler que les lectures des diodes s'effectuent généralement le plus tôt possible après l'irradiation. L'influence du traitement thermique pendant les 7 premiers jours après irradiation est présentée dans le tableau IV, où on peut constater que :

a) l'ébullition pendant 3 minutes suffit pour stabiliser le signal pour cette période donnée;

b) le traitement thermique diminue le signal, d'autant plus que le traitement est plus prolongé. On peut dire que le traitement au four apporte déjà un affaissement sensible.

Pendant la première série, un chauffage supplémentaire de toutes les diodes (témoins compris) a été effectué 78 jours après 1'irradiation. Ce traitement a induit une nouvelle diminution du signal, moins importante pour les diodes déjà traitées que pour celles non traitées. Il introduit donc un affaissement supplémentaire.

\subsection{Réponse des diodes aux neutrons du réacteur SILENE}

Les valeurs en kerma-tissu dû aux neutrons sont determinées, en tenant compte de la distance du cœur du réacteur (Medioni and Plawinski, 1997; Nurdin, 1996), à partir des valeurs de réference établies lors de la campagne d'intercomparaison des 
TABLEAU V

Réponses moyennes des diodes aux neutrons du réacteur SILENE. Average responses of diodes to the SILENE reactor neutrons.

\begin{tabular}{|c|c|c|c|}
\hline \multirow[t]{2}{*}{ Type de diode } & \multirow[t]{2}{*}{ Traitement } & \multicolumn{2}{|c|}{ Réponse moyenne, $\mathrm{mV} \mathrm{Gy}{ }^{-1}$} \\
\hline & & SILENE sous $\mathrm{Pb}$ & SII.ENE nu \\
\hline \multirow{4}{*}{$\mathrm{Si}-1$} & aucun, 7 jours après & $182,0 \pm 2,3^{(1)}$ & $170,0 \pm 4,5$ \\
\hline & ébullition & $167,0 \pm 4,8$ & - \\
\hline & four, $1 / 2$ heure, & $144,8 \pm 1,9$ & $142,8 \pm 2,6$ \\
\hline & four, 1 heure & $137,3 \pm 5,1$ & $136,6 \pm 1,3$ \\
\hline \multirow{4}{*}{$\mathrm{Si}-2$} & aucun, 7 jours après & $1320 \pm 45$ & $1139 \pm 41$ \\
\hline & ébullition & $1157 \pm 42$ & - \\
\hline & four, $1 / 2$ heure & $1042 \pm 35$ & $965 \pm 46$ \\
\hline & four, 1 heure & $1006 \pm 47$ & $981 \pm 48$ \\
\hline
\end{tabular}

(1) écart-type de la moyenne de l'ensemble des diodes traitées de la même façon et lecture unique; pour la lecture stabilisée et type Si-2, les réponses sont en moyenne de $4 \%$ plus élevées.

dosimètres de criticité (Medioni and Delafield, 1997). Elles sont pour une distance de $4 \mathrm{~m}$ du coeur et pour une production de $10^{17}$ fissions, 1,12 Gy pour l'irradiation SILENE/Pb et 1,28 Gy pour celle SILENE nu. Ces valeurs correspondent à une fluence autour de $4 \times 10^{10} \mathrm{~cm}^{-2}$ des neutrons pour SILENE nu, autour de $5 \times 10^{10} \mathrm{~cm}^{-2}$ pour $\mathrm{SILENE} / \mathrm{Pb}$.

Au vu des réponses moyennes obtenues pour les deux types de diodes, on peut montrer (voir le tableau V):

- qu'il n'y a pas de différence significative entre les valeurs obtenues pour les diodes irradiées avec ou sans fantôme BOMAB;

- que les lectures obtenues pour les deux types de diodes après le même traitement thermique sont, pour les deux spectres neutroniques du réacteur SILENE, égales.

L'ensemble des résultats presentés jusqu'à present correspondent aux réponses moyennes, c'est-à-dire aux moyennes des lectures moyennes. Pour plus de précision, nous presentons également les données des réponses individuelles. Celles-ci sont, naturellement, influencées par le choix et le nombre des diodes testées. Pour éliminer l'influence du «fading», nous ne prenons en compte que les résultats des diodes chauffées au four. Nous pouvons alors constater que:

a) le rapport maximum/minimum des sensibilités individuelles est de 1,11 pour les diodes $\mathrm{Si}-1$, et de 1,55 pour les diodes $\mathrm{Si}-2$. Ces valeurs correspondent aux réponses individuelles aux neutrons du réacteur SILENE entre: 130 et $144 \mathrm{mV} \mathrm{Gy}^{-1}$ pour les diodes Si-1, et 780 et $1210 \mathrm{mV} \mathrm{Gy}^{-1}$ pour les diodes Si-2; 
b) la réproductibilité des réponses individuelles est bonne. L'écart-type relatif pour les diodes $\mathrm{Si}-1$ varie entre 0,40 et $0,98 \%$, et entre 0,68 et $2,90 \%$ pour les diodes $\mathrm{Si}-2$. Les valeurs moyennes de l'écart-type relatif sont égales à $(0,76 \pm 0,19) \%$ pour les diodes $\mathrm{Si}-1,(1,24 \pm 0,51) \%$ pour les diodes $\mathrm{Si}-2$.

\section{Conclusions}

\subsection{Les connaissances acquises}

a) La reproductibilité des lectures de tension est bonne. Dans le cas des diodes témoins, elle est de 2 à $3 \%$, tandis que,pour les diodes irradiées, elle est d'environ de $1 \%$ pour $\mathrm{Si}-1$ et d'environ $1,5 \%$ pour $\mathrm{Si}-2$. Il faut ajouter, qu'en absolu, la reproductibilité est pour les témoins de $2 \mathrm{mV}$ (Si-1), ou de $7 \mathrm{mV}$ (Si-2). Elle est par contre plus élevée, suivant le niveau d'irradiation, pour les diodes irradiées.

b) La répétition des lectures conduit à une augmentation de la tension, pratiquement négligeable pour $\mathrm{Si}-1$, mais dont il faut tenir compte pour Si-2. Aucune différence importante n’a été observée concernant la reproductibilité des lectures uniques ou des lectures «stabilisées», ni sur l'influence du choix du mode de lecture sur d'autres caractéristiques.

c) L'homogénéité du lot des diodes Si-1 est bonne, avec un écart-type relatif $4 \%$ acceptable. On peut envisager de les utiliser sans l'étalonnage individuel. Par contre, pour les diodes Si-2, des différences de sensibilité individuelles jusqu'à $\pm 25 \%$ ont été observées.

d) La reproductibilité du comportement des diodes des deux types est satisfaisante. Une fois la sensibilité individuelle établie, les diodes permettent d'établir le kerma dû aux neutrons avec un écart-type relatif inférieur à $5 \%$. La reproductibilité du «fading» des diodes thermiquement non-traitées est du même ordre de grandeur.

e) Des données nouvelles concernant le fading des diodes et sa suppression par traitement thermique ont été acquises. L'ébullition dans l'eau pendant 3 minutes diminue son importance surtout pour les diodes $\mathrm{Si}-1$ qui sont plus petites. Le chauffage dans le four $\left(100^{\circ} \mathrm{C}\right)$ elimine pratiquement le fading pour une période d'au moins 80 jours. Il semble, toutefois, que ce traitement mène à un «annealing» du signal, qui a aussi été observé aprés le même traitement réalisé 78 jours aprés l'irradiation.

f) Les réponses des diodes testées aux neutrons du réacteur SILENE sous $\mathrm{Pb}$ et nu sont très voisines (voir aussi Spurny et al., 1995), et, également, proches de la réponse aux neutrons de ${ }^{252} \mathrm{Cf}$. Des études complémentaires sont nécessaires pour vérifier la dépendance énergétique de la réponse. 
g) L'étalonnage individuel devrait permettre de travailler à partir des valeurs de kerma suivantes :

$$
\begin{aligned}
& \text { I: } \mathrm{Si}-1\left(130 \mathrm{mV} \mathrm{Gy}^{-1}\right) ; 3 \sigma_{\text {témoins }} \sim 6 \mathrm{mV} \text {, i.e. } \mathrm{k}_{\text {seuil }} \sim 0.05 \mathrm{~Gy} \\
& \text { II: } \mathrm{Si}-2\left(1100 \mathrm{mV} \mathrm{Gy}^{-1}\right) ; 3 \sigma_{\text {témoins }} \sim 18 \mathrm{mV} \text {; i.e. } \mathrm{k}_{\text {seuil }} \sim 0.016 \mathrm{~Gy}
\end{aligned}
$$

\section{2. Études complémentaires à recommander}

a) Variation de la réponse avec le traitement thermique

Il faut optimiser le traitement thermique. En particulier, le chauffage au four pourrait être plus court, l'ébullition dans l'eau plus longue. Il faut décider d'un niveau de fading acceptable, et, donc, optimiser le temps du traitement après irradiation. Il serait aussi souhaitable de tester l'influence de la témperature ambiante sur la réponse.

b) Vérification de la réponse en fonction de l'énergie des neutrons

Il serait souhaitable de vérifier la dépendance énergetique de la réponse des diodes aux neutrons d'autres sources, comme ${ }^{252} \mathrm{Cf}$, PuBe (AmBe), SILENE et/ou un autre réacteur, etc. Simultanémment, il faut réfléchir (et décider) comment et dans quelles circonstances choisir l'étalonnage individuelle des diodes.

c) Linéarité de la réponse

Il faut vérifier la linéarité de la réponse en fonction de la dose dans la gamme des doses à mesurer.

\section{RÉFÉRENCES}

Blanc D. (1990) Les rayonnements ionisants. détection, spectrométrie, dosimétrie. Masson, Paris.

Delafield H.J., Reading A.H. (1981) Silicon diode measurements for monoenergetic neutrons and critical assemblies (HPRR and VIPER). UKAEA report AERE-M 3196 (HMSO, London).

Medioni R., Delaficld H. (1997) Criticality accident dosimetry. An international intercomparison at the SILENE reactor. Radiat. Prot. Dosim 70, $445-454$.

Medioni R., Plawinski L. (1997) communication privée.

Nurdin G. (1996) Étude de structures destinées à modifier le spectre du rayonnement autour du récteur SILENE. Note Technique $n^{\circ} 474$, DGA.

Prouza Z., Obraz O., Sopko B., Spurny F., Skubal A., Kits J., Latal F. (1989) Dosimetric parameters of a new czechoslovak neutron Si-diode. Radiat. Prot. Dosim. 28, 277-281.

Skubal A. (1981) The basic dosimetric characteristics of the czechoslovak Si-diodes with a long base and their dependence on the production technology. PhD Thesis, Czech Technical University, Prague.

Spurny F. et al. (1995), in: An intemational intercomparison of criticality accident dosimetry systems at the SILENE reactor. AEA Technology and IPSN Report HPS/TR/H, part 2, pp. 23-28.

Tournier B., Barbry F., Verrey B. (1997) SILENE. A tool for neutron dosimetry. Radiat. Prot. Dosim. 70, $345-348$. 